\title{
The role of tourniquet in myomectomy: an observational study
}

\author{
Urmi Sanyal $^{1 *}$, Shilpa Ghosh ${ }^{1}$, Hiremath P. B. ${ }^{1}$, Reshma Hiremath ${ }^{2}$
}

\begin{abstract}
${ }^{1}$ Department of Obstetrics and Gynecology, SVMCH and RC, Ariyur, Pondicherry, India
\end{abstract}
${ }^{2} \mathrm{IMO}$, Mapusa, Goa, India

Received: 06 February 2019

Accepted: 06 March 2019

*Correspondence:

Dr. Urmi Sanyal,

E-mail: urmisanyal@gmail.com

Copyright: (C) the author(s), publisher and licensee Medip Academy. This is an open-access article distributed under the terms of the Creative Commons Attribution Non-Commercial License, which permits unrestricted non-commercial use, distribution, and reproduction in any medium, provided the original work is properly cited.

\begin{abstract}
Background: Leimymoma is one of the commonest benign tumours encountered in women during the reproductive age. One quarter of these women may be symptomatic. When surgical management is indicated, myomectomy may be considered as the procedure of choice. Myomectomy can be complicated by severe intraoperative haemorrhage. One of the methods to reduce blood loss during myomectomy is the mechanical application of tourniquet.

Methods: A prospective observational single arm study was done with 24 women who underwent open myomectomy to determine the utility of tourniquet in reducing blood loss during the procedure. Women of reproductive age group, having symptomatic fibroid, not responding to medical therapy, not completed their family and with total uterine size not exceeding 20 weeks were included in the study while those with pregnancy, concomitant adenomyosis, cervical or broad ligament fibroid, bleeding diathesis were excluded. The 7-French pediatric feeding tube was used as tourniquet. Our primary end point was intra-operative blood loss. Secondary outcome measures included operative morbidity and blood transfusion rates.

Results: It was seen that application of the tourniquet considerably reduced the amount of blood loss and made resection of the myoma much easier. $83.3 \%$ patients had a blood loss less than $200 \mathrm{ml}$ and the rest between $200 \mathrm{ml}$ and $400 \mathrm{ml}$. For majority of the patients (75\%) the postoperative change of PCV was between 1 and $2 \%$.

Conclusions: The infant feeding tube form of tourniquet is cheap, safe, readily available, effectively reduces blood loss during myomectomy while not adding to the complications due to the operation.
\end{abstract}

Keywords: Myomectomy, Pediatric feeding tube, Tourniquet, Uterine fibroid

\section{INTRODUCTION}

Uterine fibroids (leiomyomata) are the most common benign tumours in women, with a lifetime prevalence of around $30 \% .^{1}$ These tumours are overgrowths of smooth muscle and connective tissue that are hormone dependent. Fibroids may be solitary, multiple or diffuse. The majority of fibroids do not cause any symptoms but one in four women with fibroids are symptomatic. ${ }^{2,3}$ The symptoms depend on the location and size of the fibroid and include heavy menstrual bleeding, pain during periods and intercourse, a dragging sensation in the lower abdomen and urinary or defecation problems. ${ }^{4}$ They can cause concern in women of reproductive age because of heavy, irregular menstrual bleeding and pain, which can have a negative impact on a woman's life and warrants intervention. $^{5}$

Treatment options include nonsurgical methods (pharmacological, uterine artery embolisation [UAE], magnetic resonance imaging [MRI]-guided focused ultrasound [MRgFUS]), minimally invasive surgery (hysteroscopic myomectomy, laparoscopic myomectomy), and open surgery (myomectomy or 
hysterectomy). The choice of treatment has to be tailored for each patient according to their wishes, the type and location of the fibroid, associated symptoms and availability of service. ${ }^{1}$

Surgical management of uterine fibroids may be required in women with severe pressure symptoms, unresponsiveness to other therapies or in large pedunculated, subserosal or submucous fibroids. Surgical treatment can be either hysterectomy or myomectomy. The size and location of the fibroid in the uterus and the desire for future fertility affects the choice of surgical procedure.

Despite the introduction of new non-surgical techniques, where fertility is to be preserved, myomectomy remains the most likely treatment option specially in case of large fibroids. Though laparoscopic myomectomy is widely practiced, myomectomy at open laparotomy is the timehonoured uterus-conserving surgical procedure of choice in low resource settings. ${ }^{6}$

Myomectomy is associated with intra-operative and postoperative complications including excessive haemorrhage, pyrexia, visceral damage, thromboembolism, conversion to hysterectomy, blood transfusions, scar dehiscence in future pregnancy and many others. ${ }^{7}$ Therefore proper haemostasis and layered closure of uterus following myomectomy are very important issues to minimize complications. Haemorrhage is a major concern in myomectomy operation. Bleeding can be prevented if dissection done through the avascular cleft or decreased with mechanical or pharmacological methods. Techniques for reducing blood loss during myomectomy include a preoperative course of GnRH analogue or Selective Progesterone Receptor Modulators (SPRM), use of vasoconstriction agents(vasopressin) and use of haemostatic tourniquets during surgery. ${ }^{8}$

Haemostatic tourniquets to reduce intra-operative bleeding have long been available but extensive data on their efficacy from controlled trials are lacking. This study assesses its efficacy and safety of the use of tourniquet to reduce blood loss and its complications (if any).

\section{METHODS}

This is a prospective single arm interventional study. It was conducted from June 2016 to December 2017. The study was done after taking the permission from the local ethical committee in accordance with the ethical standards. A total number of 24 women were recruited and planned for open myomectomy.

\section{Inclusion criteria}

- Women of reproductive age group, having symptomatic fibroid
- Not responding to medical therapy

- Women who have not completed their family

- Total uterine size not exceeding 20 weeks.

\section{Exclusion criteria}

- Pregnancy, concomitant adenomyosis, cervical or broad ligament fibroid, bleeding diathesis, cases unfit for surgery or anaesthesia.

Diagnosis of uterine fibroid was based on clinical examination and ultrasound findings. Women mainly came with complains of abnormal uterine bleeding (e.g. menorrhagia, intermenstrual bleeding) not responding to medical management. Other complains were abdominal mass, infertility, abdominal pain, dysmenorrhoea and some patients also presented with pressure symptoms on the bladder and bowel.

After thorough assessment of all the parameters women were included in the study. It was decided that open myomectomy will be performed for all patients included in the study and tourniquet will be applied during the procedure to reduce intra operative blood loss. Since present study is done in a low-resources setting, most patients are from lower socio-economic classes and are not affordable for expensive drugs like vasopressin. Also, our institution is a medical college, so authors wanted to give their postgraduate trainees an idea about the effect of tourniquet placement to reduce blood loss during myomectomy.

Intra-operatively for majority of the patient's abdomen was opened by a low transverse incision. Pfannenstiel incision was found to be adequate for myomectomy on a small fibroid. Authors preferred the Maylard incision in case of larger uterus more than 12 weeks. After assessing the anatomy of the uterus and the operability of the fibroid, plan of myomectomy was confirmed. The uterus was exteriorized, and the bowels packed away using two large wet swabs. Authors tried to use a single midline anterior incision on the uterus whenever possible. Initially, a small opening was made in an avascular space in the broad ligament on either side of the uterine isthmus just lateral to the uterine vessels. A 7-French pediatric feeding tube is looped around the upper cervix through the opening in the broad ligament, $1 \mathrm{~cm}$ above the uterovesical fold (Figure 1). Then the procedure of myoma dissection was done (Figure2). It was seen that application of the tourniquet considerably reduced the amount of blood loss and made resection of the myoma much easier (Figure 3). Blood loss was assessed intraoperatively by weighing swabs and measuring blood collected by suction and postoperatively by changes in PCV). The primary end point was intra-operative blood loss. Secondary outcome measures included blood transfusion rates and operative morbidity (return to theatre, pyrexia $>38 \mathrm{C}$ on more than two occasions 4 hours apart, bowel obstruction, wound infection, wound 
haemorrhage, chest infection and venous thromboembolism).

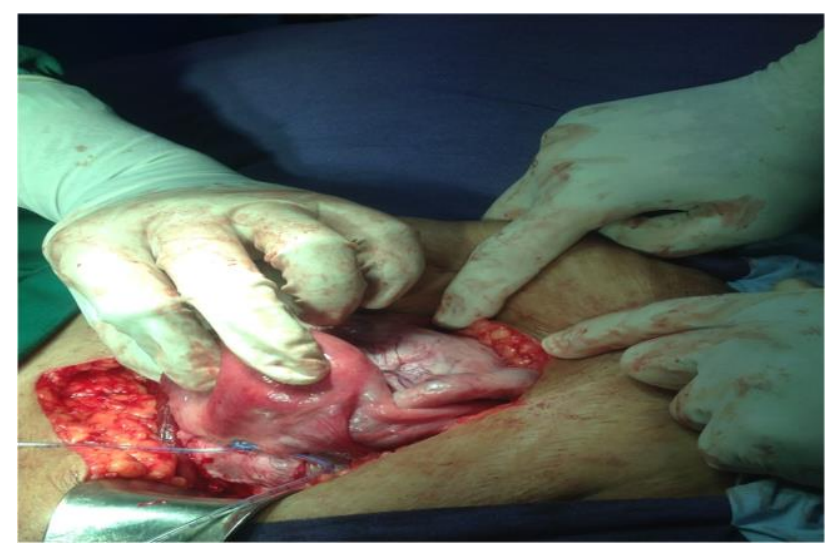

Figure 1: Tourniquet tied at the cervico-isthmic junction.

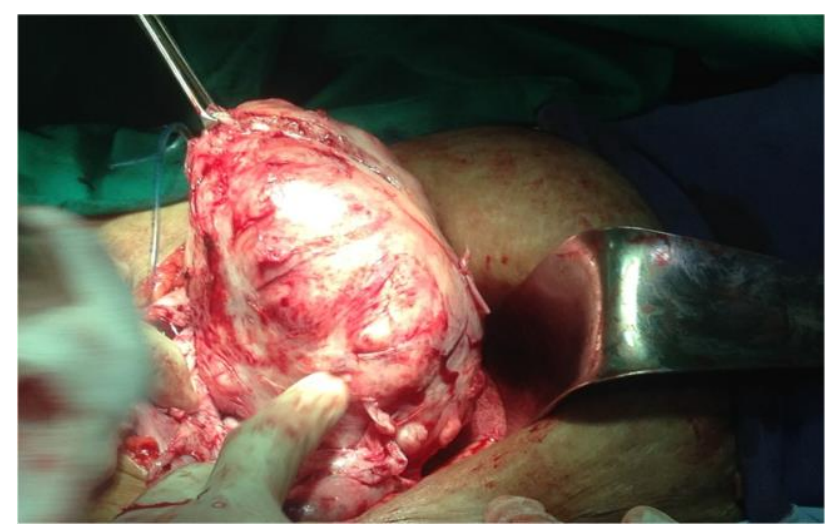

Figure 2: Enucleation of fibroid done.

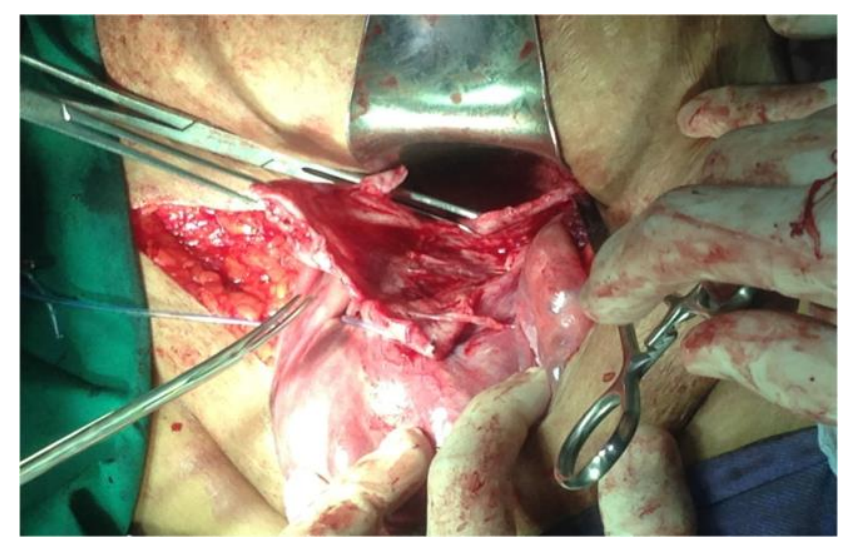

Figure 3: Relatively blood less field due to application of tourniquet.

\section{RESULTS}

Table 1 and Table 2 give the details about the 24 women who gave consent for open myomectomy. $33.33 \%$ belonged to the age group of 25-29 years while $29.16 \%$ belonged between 30-34 years of age. One patient was more than 45 years old. Majority of the women (70.8\%) were nulliparous.

Table 1: Distribution of women according to age.

\begin{tabular}{|l|l|}
\hline Age & Number (Percentage) \\
\hline$<25$ years & $1(4.17)$ \\
\hline 25-29 years & $8(33.33)$ \\
\hline 30-34 years & $7(29.16)$ \\
\hline 35-39 years & $3(12.5)$ \\
\hline $40-44$ years & $4(16.67)$ \\
\hline$>45$ years & $1(4.17)$ \\
\hline
\end{tabular}

Table 2: Distribution of women according to parity.

\begin{tabular}{|l|l|}
\hline Parity & Number (Percentage) \\
\hline 0 & $17(70.8)$ \\
\hline $1-4$ & $5(20.8)$ \\
\hline $5-7$ & $2(8.4)$ \\
\hline
\end{tabular}

Table 3 shows the variety of symptoms with which the patients presented. The most common symptom was abdominal mass in $25 \%$ cases followed by menorrhagia in $20.83 \%$ cases. Patients presented with dysmenorrhea in $16.67 \%$ cases. Irregular vaginal bleeding and vaginal discharge were present in $8.33 \%$ cases each and urinary symptoms were present in $4.17 \%$ cases. 1 patient presented with infertility while another had a history of recurrent pregnancy loss.

Table 3: Distribution of women according to clinical presentation.

\begin{tabular}{|l|l|}
\hline Symptoms & Number (Percentage) \\
\hline Abdominal mass & $06(25)$ \\
\hline Menorrhagia & $05(20.83)$ \\
\hline Infertility & $01(4.17)$ \\
\hline Abdominal pain & $02(8.33)$ \\
\hline Dysmenorrhoea & $04(16.67)$ \\
\hline Irregular vaginal bleeding & $02(8.33)$ \\
\hline Urinary symptoms & $01(4.17)$ \\
\hline Vaginal discharge & $02(8.33)$ \\
\hline Recurrent abortion & $01(4.17)$ \\
\hline
\end{tabular}

Table 4 shows the distribution of the fibroids according to their location in the uterus. Majority i.e. in 20 cases $(83.3 \%)$ it was intramural while it was subserous in 3 $(12.5 \%)$ cases and submucous in 1 case.

Table 4: Distribution of women according to the location of fibroid.

\begin{tabular}{|l|l|}
\hline Location of fibroid & Number (Percentage) \\
\hline Intramural & $20(83.3)$ \\
\hline Subserous & $3(12.5)$ \\
\hline Submucosal & $1(4.2)$ \\
\hline
\end{tabular}

Table 5 shows distribution of patients according to preoperative hemocrit status. Majority of the patients i.e. 
$48.2 \%$ had hemocrit between $30-33 \% .3$ patients $(14.2 \%)$ had hemocrit less than 25. Table 6 gives us an account of the average blood loss during surgery after application of tourniquet. $83.3 \%$ patients had a blood loss less than $200 \mathrm{ml}$ and the rest between $200 \mathrm{ml}$ and $800 \mathrm{ml}$.

Table 5: Distribution of women according to preoperative PCV.

\begin{tabular}{|l|l|}
\hline Pre-op PCV & Number (Percentage) \\
\hline$<25$ & $3(14.8)$ \\
\hline $25-29$ & $3(14.8)$ \\
\hline $30-33$ & $11(48.2)$ \\
\hline 34 & $7(22.2)$ \\
\hline
\end{tabular}

Table 6: Distribution of patients according to intraoperative blood loss.

\begin{tabular}{|l|l|}
\hline Intra-operative blood loss $(\mathrm{ml})$ & No. of patients $(\%)$ \\
\hline$<200$ & $20(83.3)$ \\
\hline $200-800$ & $4(16.7)$ \\
\hline$>800$ & 0 \\
\hline
\end{tabular}

Table 7 shows d change of PCV following myomectomy. For majority of the patients $(75 \%)$ the postoperative change of PCV is between 1 and $2 \%$.

Table 8 shows the incidence of postoperative complications among the patients. Febrile complications were most common and occurred among $25 \%$ of the patients.

Table 7: Postoperative change (drop) of packed cell volume (PCV).

\begin{tabular}{|l|l|}
\hline Change of PCV & No. of patients $(\%)$ \\
\hline$<1$ & $2(8.3)$ \\
\hline $1-2$ & $18(75)$ \\
\hline $2-3$ & $4(16.7)$ \\
\hline$>3$ & 0 \\
\hline
\end{tabular}

Table 8: Postoperative morbidity.

\begin{tabular}{|l|l|}
\hline Postoperative morbidity & No. of patients (\%) \\
\hline Blood transfusion & $2(8.3)$ \\
\hline Return to theatre & 0 \\
\hline Febrile complications & $6(25)$ \\
\hline Wound infections & $2(8.3)$ \\
\hline
\end{tabular}

Overall in present study, there were no major postoperative complications like return to theatre for relaparatomy or hysterectomy due to excessive bleeding. 2 patients required blood transfusion postoperatively. Intraoperatively also only one patient had bleeding in the broad ligament during the tourniquet placement which was successfully controlled with sutures and cautery. One infertile patient conceived. All patients were satisfactorily relieved of the symptoms.

\section{DISCUSSION}

In our setting, for the foreseeable future, the main stay of treatment for symptomatic fibroid will have to be surgical, particularly myomectomy. The high cost of medical therapy and the associated side effects of the long-term use, the relative unavailability of the radiological options and the need to preserve the uterus for fertility and femininity virtually assure this. Also, the majority of women in present study had their age below 40 years and are nulliparous, so it is of utmost importance to achieve cure without loss of function or deformity.

The goal of a myomectomy operation is to remove all the visible and accessible myomas and then reconstruct the uterus. Present study involved open route of myomectomy by laparotomy. At present a large number of teams use laparoscopic myomectomy to remove subserous and intramural myomas. However, laparoscopic route is still the subject of considerable debate: in particular the technique is reputed to be technically difficult, long, and to involve a high risk of conversion to laparotomy. ${ }^{9}$ Dissection of the myoma may be difficult because the cleavage plane is impossible to find, provoking bleeding. The haemorrhage may be abundant. The hysterotomy may be difficult to close because of its length, its location or bleeding. As conversion to laparotomy is more time and cost consuming than using laparotomy from the outset, this risk might be an obstacle for diffusion of the procedure, in particular in non-teaching hospitals. However, laparoscopic myomectomy is associated with less subjectively reported postoperative pain, lower postoperative fever and shorter hospital stay. ${ }^{10}$

Myomectomy is notorious for inherent dangers and potentials for complications. With the advent of safer surgical techniques, potent antibiotics and availability of safe and efficient blood transfusion services, the morbidity and mortality following myomectomy has reduced over the ages. However, the risk of severe haemorrhage still remains. Clinical experience and pooled results of numerous studies suggest that myomectomy can result in considerable blood loss. ${ }^{11,12}$ Its mainly due to the increased vascularity of the uterus and the need for complete dissection of the mass to prevent recurrences. Hemostasis at the time of surgery is an important issue for the success of the operation and patient's recovery. Several techniques have been devised to reduce the loss of blood at surgery. Procedures include the intra-operative use of Bonney's clamp or tourniquet, injection of vasopressin intra-myometrially, pre-operative administration of $\mathrm{GnRH}$ analogue or misoprostol. ${ }^{13}$

The tourniquet is by far the cheapest and most accessible for our environment. The infant's feeding tube or the conventional Foley's urethral catheter is easily adapted for this purpose. 
The main blood supply of the uterus is from the uterine arteries, which pass through the cardinal ligament at level of the cervico-uterine junction. The purpose of application of tourniquet is to temporarily occlude the blood supply to the uterus and consequently to the fibroid, reducing the vascularity of the lesion. Early proponents of myomectomy focused on methods to temporarily occlude uterine blood flow to control hemorrhage and provide a bloodless operative field. One of the earliest methods was simply to have an assistant grasp the broad ligaments firmly with each hand during myomectomy to impede blood flow through the uterine vessels. In the 1920s, Victor Bonney introduced a specially designed clamp that was placed around the uterine vessels and the round ligaments. ${ }^{14}$ The ovarian vessels were occluded with ring forceps. Using this technique, he was able on one occasion to remove more than 200 myomata from a single uterus. Rubin, in 1938, was the first to use an elastic rubber tourniquet through the broad ligament, encircling the cervix and occluding the uterine vessels during myomectomy. Rubber-shod clamps applied to the broad ligaments have also been used to occlude the uterine vessels and control bleeding. ${ }^{15}$

Intra-operative vasopressin is also widely used but it is expensive and may predispose to severe complications. Vasopressin should not be used in patients with vascular disease, especially the coronary artery diseases. Inadvertent intravascular injection can cause anginal pain; larger doses can cause myocardial infarction. Water intoxication can also occur as a result of the antidiuretic effect of vasopressin. ${ }^{16-18}$ Late postoperative bleeding also reported with the use of vasopressin. Because of the short half-life of vasopressin, the hemostatic effect is observed only for 20 to 30 minutes and should be over before the incision closure is started. Some also claim that vasopressin simply delays bleeding, gives a false sense of security, and is not particularly effective for larger myomata and very extensive myomectomies. ${ }^{15}$ These are also the reason that prompted us to study the use of simple but effective mechanical tourniquet for the control of bleeding during myomectomy.

GnRH analogues are expensive and are associated with menopausal side effects with prolonged use. Although they reduce the size of the fibroid masses, they have been shown not to affect the amount of blood loss at surgery. ${ }^{19}$ In addition, they cause a blurring of the planes of the pseudo-capsule and subsequently makes healing of the mass difficult and the smaller masses disappear before surgery only to regrow on cessation of the treatment.

The infant feeding tube form of tourniquet as shown in present study is very effective with significant reduction in blood loss. It has been proved in multiple studies that application of tourniquets to the uterine vessels during myomectomy significantly reduces intraoperative blood loss, the need for blood transfusion and postoperative morbidity. Our result agrees with the findings of Kongnuyi and Wiysonge where significant reduction in blood loss was also noted with tourniquet usage. ${ }^{20}$ Similar findings were observed in the study by JI Ikechebelu et al where use of tourniquet was compared with non-use. ${ }^{21}$

In present study, the reduction in blood loss on using tourniquet led to a reduced need for peri-operative or postoperative blood transfusion. This translated to reduced exposure to the complications of blood transfusion and improved postoperative recovery. A clear operating field is another important advantage gained from the use of the tourniquet leading to an easier and faster dissection of myoma and subsequent reduced intra operative complications. The virtually bloodless will allowed us the opportunity to remove every visible piece of fibroid.

Once the tourniquets are tightened in place, the myomectomy should proceed expeditiously to prevent ischemic damage to the uterus, tubes, and ovaries due to prolonged devascularization. The length of time for which the pelvic structures can be without blood flow before irreversible damage occurs is unknown. However, authors did not release the tourniquets until the myomectomy was complete (usually within an hour) and have not experienced any adverse events. Other authors also agree that intermittent release of the tourniquets is unnecessary. ${ }^{15}$ However, because the potential for injury does exist, tourniquet time should be monitored and kept to a minimum. The tourniquets were not tightened until the surgical team is ready to perform the myomectomy. Intermittent release of the tourniquets can be considered if the operating time becomes excessive.

After reconstruction of the uterus is complete, the determination of adequate hemostasis in the uterus cannot be made until all the tourniquets are released and the blood pressure has returned to normal. Sometimes additional sutures are required for hemostasis. Before the abdomen is closed, the small holes in the broad ligament are sometimes repaired with figure-of-eight sutures.

\section{CONCLUSION}

The infant feeding tube form of tourniquet is cheap, safe, readily available, effectively reduces blood loss during myomectomy while not adding to the complications due to the operation. A criticism of uterine tourniquets is that they are traumatic to pelvic structures. Our experience, to the contrary, is that soft plastic tubes used as tourniquets are quite atraumatic. In present study, no injuries attributable to these tourniquets have occurred. However, it is essential to highlight that though tourniquet proved essential to control bleeding during myomectomy, it cannot substitute for good surgical techniques. Adherence to basic principles of surgery was essential for good outcome in present study. Perhaps the most important of these was careful planning of the uterine incisions. Authors aimed towards a minimal number of incisions. Authors always attempted removal of all leiomyomata 
through a single incision in the anterior uterine corpus. Authors tried to stick to midline incision when feasible to avoid the vascular areas of the uterus and broad ligaments laterally. Even intramural fibroids in the posterior uterine were removed through anterior incisions. This is because posterior uterine incisions lead to adhesions which likely involve the tubes and ovaries as well. Incisions in the posterior uterine wall was necessary, however, if posterior sub-serous tumors are being removed.

Funding: No funding sources

Conflict of interest: None declared

Ethical approval: The study was approved by the Institutional Ethics Committee

\section{REFERENCES}

1. Younas K, Hadoura E, Majoko F, Bunkheila A. A review of evidence-based management of uterine fibroids. TObstet Gynaecol. 2016;18(1):33-42.

2. Parazzini F. Risk factors for clinically diagnosed uterine fibroids in women around menopause. Maturitas. 2006;55(2):174-9.

3. Okolo S. Incidence, aetiology and epidemiology of uterine fibroids. Best Pract Res Clinic Obstet Gynaecol. 2008;22(4):571-88.

4. Zimmermann A, Bernuit D, Gerlinger C, Schaefers M, Geppert K. Prevalence, symptoms and management of uterine fibroids: an international internet-based survey of 21,746 women. BMC Women's Health. 2012;12(1):6.

5. Ryan GL, Syrop CH, Van Voorhis BJ. Role, epidemiology, and natural history of benign uterine mass lesions. Clinic Obstet Gynecol. 2005;48(2):312-24.

6. Manyonda I, Sinthamoney E, Belli AM. Controversies and challenges in the modern management of uterine fibroids. BJOG: Int J Obstet Gynaecol. 2004;111(2):95-102.

7. Paul GP, Naik SA, Madhu KN, Thomas T. Complications of laparoscopic myomectomy: a single surgeon's series of 1001 cases. Aus NZ J Obstet Gynaecol. 2010;50(4):385-90.

8. Kongnyuy EJ, Wiysonge CS. Interventions to reduce haemorrhage during myomectomy for fibroids. Cochrane Database Syst Rev. 2014;8:CD005355.

9. Dubuisson JB, Fauconnier A, Fourchotte V, BabakiFard K, Coste J, Chapron C. Laparoscopic myomectomy: predicting the risk of conversion to an open procedure. Human Reproduct. 2001;16(8):1726-31.

10. Chittawar PB, Franik S, Pouwer AW, Farquhar C. Minimally invasive surgical techniques versus open myomectomy for uterine fibroids. Cochrane Database of Systematic Reviews. 2014(10).

11. Alexander W. M Press and Circular, 1898; 66:349.

12. Lock FR. Multiple myomectomy. Am J Obstet Gynecol 1969;104(5):642-50.

13. Akinyemi O, Adewoye BR, Fakoya TA. Uterine Fibroids; a review. Niger J Med. 2004:13(4):318-29.

14. Bonney V. The technique and results of myomectomy. Lancet 1931;220(5604):171-7.

15. Thompson JD, Rock JA. Leiomyomata uteri and myomectomy. In: ThompsonJD, RockJA,editors. TeLinde's Operative Gynaecology. Philadelphia: Lippincott-Raven Publishers, 1997:731-770.

16. Talandi T, Beique F, Kimia M. Pulmonary oedema, a complication of local injection of vasopressin at laparoscopy. Fertil Steril. 1996:(66):478-80.

17. Martin JD, Shenk LG. Intraoperative myocardial infarction after paracervical vasopressin infiltration. Anesthes Anal. 1994;79(6):1201-2.

18. Nezhat F, Admon D, Nezhat CH, Dicorpo JE, Nezhat C. Life-threatening hypotension after vasopressin injection during operative laparoscopy, followed by uneventful repeat laparoscopy. J Am Associat Gynecol Laparoscop. 1994;2(1):83-6.

19. Vercellini P, Trespìdi L, Zaina B, Vicentini S, Stellato G, Crosignani PG. Gonadotropin-releasing hormone agonist treatment before abdominal myomectomy: a controlled trial. Fertil and Steril. 2003;79(6):1390-5.

20. Kongnyuy EJ, Wiysonge CS. Interventions to reduce haemorrhage during myomectomy for fibroids. Cochrane Database of Systematic Reviews. 2014;(8): CD005355.

21. JI Ikechebelu, CO Ezeama, Obiechina NJA. The use of tourniquet to reduce blood loss at myomectomy. Nigerian J Clinic Pract. 2010;13(2):154-8.

Cite this article as: Sanyal U, Ghosh S, Hiremath $\mathrm{PB}$, Hiremath R. The role of tourniquet in myomectomy: an observational study. Int J Reprod Contracept Obstet Gynecol 2019;8:1610-5. 